\title{
Neutrino induced showering from the Earth
}

\author{
Daniele Fargion* \\ Physics Department and INFN, University "La Sapienza", P.le A. Moro,2, 00185 \\ ROME, ITALY \\ E-mail: 'daniele.fargion@roma1.infn.it'
}

Abstract: Ultra High Energy, UHE, Neutrino Astronomy should be soon tested looking toward the Earth. At present High Energy Neutrino Astronomy is searched by AMANDA, ANTARES underground detectors looking for its consequent unique muons secondary track. We suggest a higher energy Tau Neutrino Astronomy based on Horizontal and Upward Tau Air-Showers escaping from the Earth. These Tau air-showers greatly amplifies the single tau track by an abundant secondary tail ( billions of electron pairs, gamma and tens of millions muon bundles) spread in huge areas (kilometer size) easily observable (even partially) from high mountains, balloon or satellite array detectors. Possible early evidence of such a New Neutrino UPTAUs or HORTAUs (Upward or Horizontal Tau Air-Showers) Astronomy may be already found in rare BATSE gamma records of brief up-going gamma showers named Terrestrial Gamma Flashes (TGF). The TGF features, energy and arrival clustering are well tuned to upward tau air-showers. Future confirmation of the Neutrino Tau Astronomy must be found in detectors as EUSO, seeking for Upward-Horizontal air-shower events: indeed even minimal, guaranteed, GZK neutrino fluxes may be observed if EUSO threshold reaches $10^{19} \mathrm{eV}$ or lower energies by enlarging its telescope size.

\section{From Cosmic Rays to Ultra High Energy Tau Neutrinos Astronomy}

Nature, for unknown reasons or because inflation in early Universe, did not provide (much or at all) magnetic monopoles in our Universe. Their absence is proved by the existence of coherent large scale structure of magnetic fields near planets, stars and galactic spaces (the so called Parker limit). Cosmic Rays, because of their main nucleon and nuclei nature, are charged and consequently they are easily bent by solar, galactic and extra-galactic magnetic fields. Therefore Cosmic Rays are reaching our terrestrial atmosphere after a long random walk by Lorents forces, smeared and nearly isotropic with no astronomical legacy.

\footnotetext{
${ }^{*}$ Speaker.
} 
Astronomy, on the contrary, is based on the direct, undeflected radiation from astronomical source to the observer. The neutral messengers which we did exploit in Astronomy during last three centuries were mainly photons in the optical range. Since Maxwell discovers and along last century the whole electromagnetic spectrum was opened to Astronomy. Radio, Infrared, X and gamma astronomy made vivid in new colors hidden sides of our sky. Photons are not the unique neutral particle. Indeed in the last 30 years also neutrino astronomy arose and proved fruit-fully in discovering solar and supernova neutrino astronomy at low $(\mathrm{MeV})$ energies. Also at the extreme energy edges, Ultra High Energy Cosmic Ray (UHECR) may offer a New Particle Astronomy because, even they are charged, their extreme rigidity makes them almost un-bent by galactic and extra-galactic magnetic fields. Therefore UHECR, even charged, fly nearly straight from their birth-place to us offering a New Particle Astronomy. These UHECR $\left(E_{U H E C R} \geq 10^{19} \mathrm{eV}\right)$ are numerous and they are already reaching hundreds of data records. Moreover this UHECR astronomy is bounded (by primordial photon drag, the well known Greisen, Zatsepin, Kuzmin (GZK) cut-off

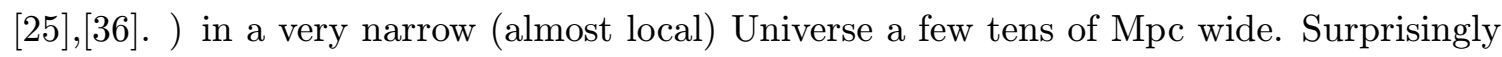
we did't find yet in present UHECR arrival maps any corresponding nearby known galactic

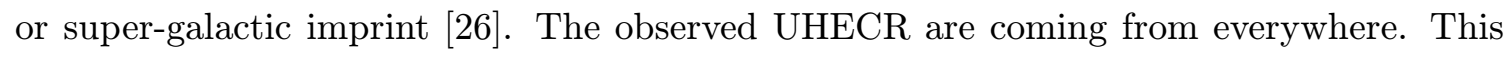
isotropy call naturally for a cosmic link, whose distances are well above the narrow GZK radius cut-off. Moreover observed UHECR clustering in groups strongly suggest compact sources (as AGN or BL Lacs beaming Jets) respect to any homogeneous and isotropic halo made by primordial topological defects. Recent evidence for a dozen or more BL

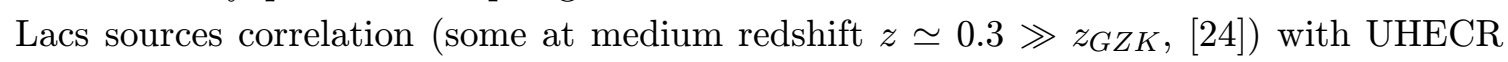
clustered events are giving support (with their over all isotropy [i2 $2 \bar{b}]$ ) to a cosmic origin for UHECR sources [i2 $\overline{2}]$. This is the so-called GZK paradox that may find a solution by neutrino with a light mass, in the so called Z-Burst model. Indeed UHE Relic neutrinos with a light mass may play a role as calorimeter for UHE neutrinos from cosmic distances

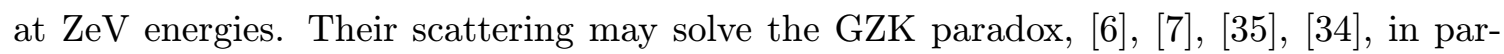

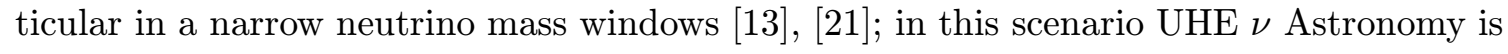
not just a consequence but itself the cause of UHECR signals. There are other additional solution to GZK paradox based on extreme extragalactic magnetic fields, new hadronic physics or hypothetical decay of primordial topological relics; we shall not address here to these models for lack of space; anyway large neutrino secondaries or primaries fluxes must also arise $[\overline{2} \bar{z}]$ in most models. Indeed the same UHECR constrained by GZK cut off must produce abundantly by pion decays the so called GZK (or cosmogenic) neutrino secondaries at a fluence at least comparable with most penetrating UHECR. Therefore there must be a minimal underlying neutrino astronomy nearly as large as observed GZK cosmic ray level (at energy $E \leq 10^{19} \mathrm{eV}$ ). In order to solve the GZK puzzles it may be necessary to trace their linked UHE neutrinos either primary (at much higher fluxes) by Z-Showering or Z-Burst model or, for GZK neutrinos at least as secondaries of the same UHECR. To test this idea we need to open an independent UHE neutrino Astronomy on Earth. Different muon track detectors, cubic $\mathrm{km}^{3}$ in underground may be reveal them at PeVs energies. Nevertheless UHE neutrino tau may interact on Mountains or better on Earth crust, at a huge concrete calorimeter, leading to Tau Air-Showers, probing easily both (secondary 
and/or primary) UHE neutrino astronomy at GZK energies. Present article review the problems and the experimental prospects on Mountains, planes detectors quota; we also reconsidered events measured by satellites, like past BATSE, present gamma satellite INTEGRAL, future EUSO experiments. The recent signals in BATSE terrestrial gamma flashes maybe indeed the first evidence for these new UHE neutrino Astronomy upcoming from Earth crust at PeV-EeV energies, leading to UPTAUs and HORTAUs showers. Indeed in last Fig. $\overline{T_{1}}$ we summirized the two consequent Flux signal derived by the TGF event rate data in BATSE (1991-2000) experiment, normalized for the estimated BATSE thresholds. These preliminary result may be a useful reference estimate for PeV-EeV neutrino fluxes and apparently they are well consistent with Z-Showering model for a relic mass within the

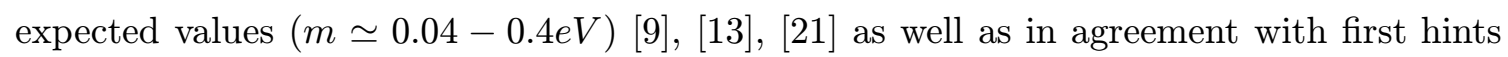

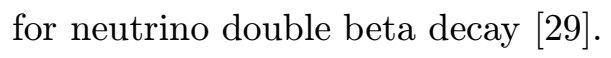

Let us remind that the restrictions on any astronomy are related to a the messenger interactions with the surrounding medium on the way to the observer. While Cosmic Rays astronomy is severely blurred by random terrestrial, solar, galactic and extragalactic magnetic lenses, the highest $\gamma$ ray astronomy (above tens $\mathrm{TeV}$ ) became more or less blind because of photon-photon opacity (due to electron pair production) at different energy windows. Indeed the Infrared- $\mathrm{TeV}$ opacity as well as a more severe Black Body Radiation, $\mathrm{BBR},($ at $2.75 \mathrm{~K}) \mathrm{-PeV}$ cut-off are bounding the $\mathrm{TeV}-\mathrm{PeV} \gamma$ ray astronomy in very nearby cosmic ( or even galactic) volumes. Therefore rarest $\mathrm{TeV}$ gamma signals are at present the most extreme trace of High Energy Astronomy. We observe copious cosmic rays at higher $\left(\gg 10^{15} \mathrm{eV}\right)$ energies almost isotropically spread by galactic and cosmic magnetic fields in the sky. Let us remind, among the $\gamma \mathrm{TeV}$ discoveries, the signals of power-full Jets blazing to us from Galactic (Micro-Quasars) or extragalactic edges (BL Lacs). At PeV energies astrophysical $\gamma$ cosmic rays should also be presented, but, excluding a very rare and elusive Cyg $X 3$ event, they have not being up date observed; only upper bounds are known at $\mathrm{PeV}$ energies. The missing $\gamma \mathrm{PeV}$ astronomy, as we mentioned, are very probably absorbed because of their own photon interactions (electron pairs creation) at the source environment and/or along the photon propagation into the cosmic Black Body Radiation (BBR) and/or into other diffused background radiation. Unfortunately $\mathrm{PeV}$ charged cosmic rays, easily bend and bounded in a random walk by Galactic magnetic fields, loose their original directionality and their astronomical relevance; their tangled trajectory resident time in the Galaxy is much longer $\left(\geq 10^{3}-10^{5}\right)$ than any linear neutral trajectory, as in the case of gamma rays, making the charged cosmic rays more probable to be observed by nearly a comparable length ratio. However astrophysical UHE neutrino signals in the wide range $10^{13} \mathrm{eV}-10^{19} \mathrm{eV}$ (or even higher GZK energies) are unaffected by any radiation cosmic opacity and may easily open a very new exciting window toward Highest Energy sources. Being weakly interacting the neutrinos are an ideal microscope to deeply observe in their accelerator (Jet,SN,GRB, Mini Black Hole) cores they do not experience any strong self opacity as the case of photon. Other astrophysical $\nu$ sources at lower energies $\left(10^{8} \mathrm{eV}\right.$ $10^{12} \mathrm{eV}$ ) should also be present, at least at EGRET fluence level, but their signals are very weak and probably drowned by the dominant diffused atmospheric $\nu$, secondaries of muon secondaries, produced as pion decays by the same charged (and smeared) UHE cosmic rays 


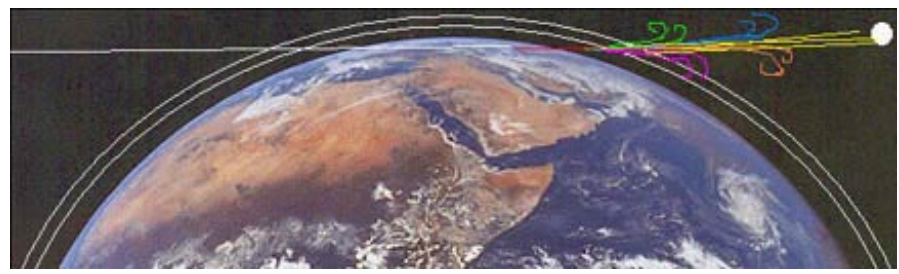

Figure 1: Horizontal Upward Tau Air-Shower (HORTAUS) originated by UHE neutrino skimming the Earth: fan-like jets due to geo-magnetic bending shower at high quota $(\sim 23-40 \mathrm{~km})$ : they may be pointing to an orbital satellite detector. The Shower tails may be also observable by EUSO just above it.

(while hitting terrestrial atmosphere): the so called diffused atmospheric neutrinos. Indeed a modulation of the atmospheric neutrinos signal and recent test from nuclear plants have inferred the first and ultimate conclusive evidence for a neutrino mass and for a neutrino

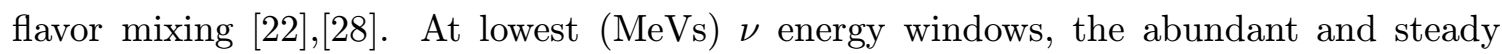
solar neutrino flux, (as well as the prompt, but rare, neutrino burst from a nearby SuperNovae (SN 1987A)), has been, in last twenty years, successfully explored, giving support to neutrino flavour mixing and to the neutrino mass reality. More recent additional probes of solar neutrino flavour mixing and reactor neutrino disappearance are giving a robust ground to the neutrino mass existence [4]-

\section{High Energy Tau Showering on top Earth Atmosphere}

While longest $\mu$ tracks in $\mathrm{km}^{3}$ underground detector have been, in last three decades, the main searched UHE neutrino signal, Tau Air-showers by UHE neutrinos generated in Mountain Chains or within Earth skin crust, see Fig.'i up to GZK $\left(>10^{19} \mathrm{eV}\right)$ or upward Fig.

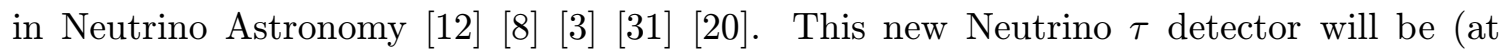
least) complementary to present and future, lower energy, $\nu$ underground $\mathrm{km}^{3}$ telescope projects (from AMANDA, Baikal, ANTARES, NESTOR, NEMO, IceCube). In particular Horizontal Tau Air shower may be naturally originated by UHE $\nu_{\tau}$ at GZK energies crossing

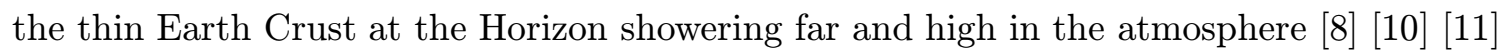

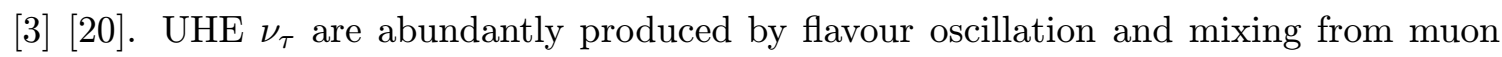
(or electron) neutrinos, because of the large galactic and cosmic distances respect to the neutrino oscillation ones (for already known neutrino mass splitting). Therefore EUSO may observe many of the above behaviours and it may constrains among models and fluxes and it may also answer open standing questions. We will briefly enlist, in this presentation, the main different signatures and rates of UHECR versus UHE $\nu$ shower observable by EUSO at $10 \%$ duty cycle time within a 3 year record period, offering a more accurate estimate of their signals [ị $\underline{\underline{9}} \bar{i}$.

Let us first consider the last kind of Upward $\tau$ signals due to their interaction in Air or in Earth Crust (UPTAUs)Fig. 3. . The Earth opacity will filter mainly $10^{14} \div 10^{16} \mathrm{eV}$

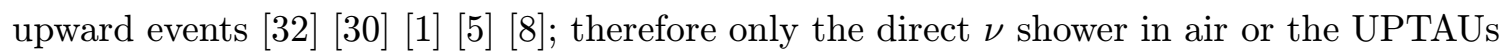




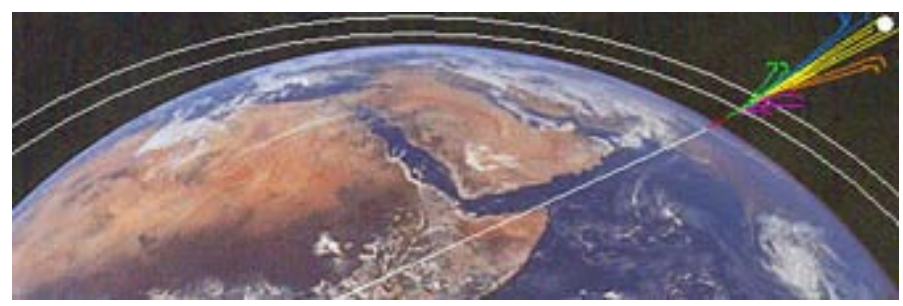

Figure 2: A very schematic Upward Tau Air-Shower (UPTAUs) and its open fan-like jets due to geo-magnetic bending at high quota $(\sim 20-30 \mathrm{~km})$. The gamma Shower may be pointing to an orbital detector. Its vertical Shower tail may be spread by geo-magnetic field into a thin eight-shape beam observable by EUSO as a small blazing oval (few dot-pixels) aligned orthogonal to the local magnetic field .

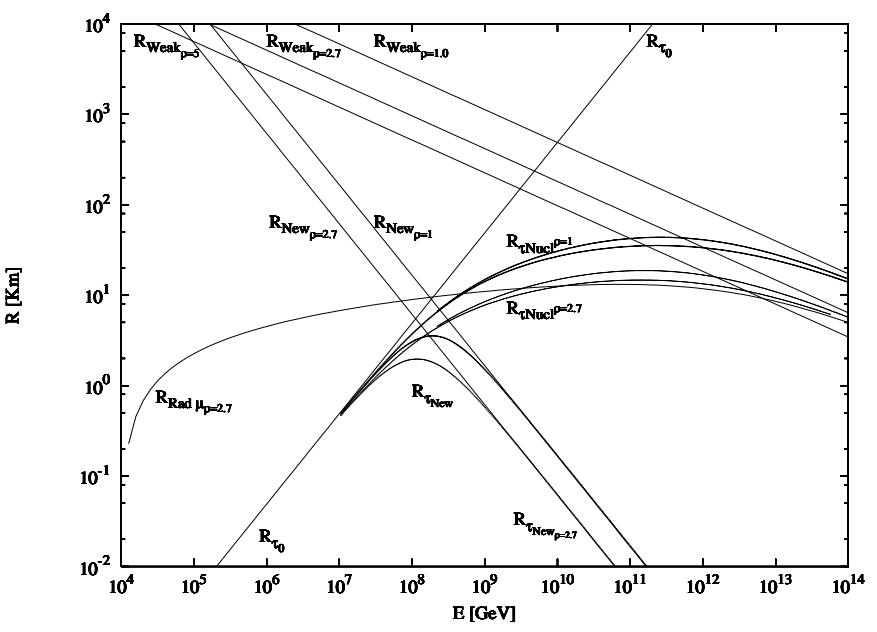

Figure 3: Lepton $\tau$ (and $\mu$ ) Interaction Lengths for different matter densities: $R_{\tau_{o}}$ is the free $\tau$ length, $R_{\tau_{N e w}}$ is the New Physics TeV Gravity interaction range at corresponding densities, $R_{\tau_{N u c l} \cdot \rho}$ , is the combined $\tau$ Ranges keeping care of all known interactions and lifetime and mainly the photo-nuclear interaction. There are two slightly different split curves (for each density) by two comparable approximations in the interaction laws. Note also the neutrino interaction lenghts above lines $R_{W e a k \rho}=L_{\nu}$ due to the electro-weak interactions at corresponding densities.

around $3 \mathrm{PeVs}$ will be able to flash toward EUSO in a narrow beam $\left(2.5 \cdot 10^{-5}\right.$ solid angle) jet blazing apparently at $10^{19} \div 10^{20} \mathrm{eV}$ energy. The shower will be opened in a fan like shape and it will emerge from the Earth atmosphere spread as a triplet or multi-dot signal aligned orthogonal to local terrestrial magnetic field. This rare multi-dot polarization of the outcoming shower will define a characteristic signature easily to be revealed. Its opening will be orthogonal to the magnetic field lines at that particular geological area. However the effective observed air mass by EUSO is not $10 \%$ (because duty cycle) of the inspected air volume $\sim 150 \mathrm{~km}^{3}$, but because of the narrow blazing shower cone it corresponds to only to $3.72 \cdot 10^{-3} \mathrm{~km}^{3}$. The target volume increases for upward neutrino Tau interacting vertically in Earth Crust in last matter layer (either rock or water), see Fig. ${ }_{3}$, in this case the effective target mass is (for water or rock) respectively $5.5 \cdot 10^{-2} \mathrm{~km}^{3}$ or 


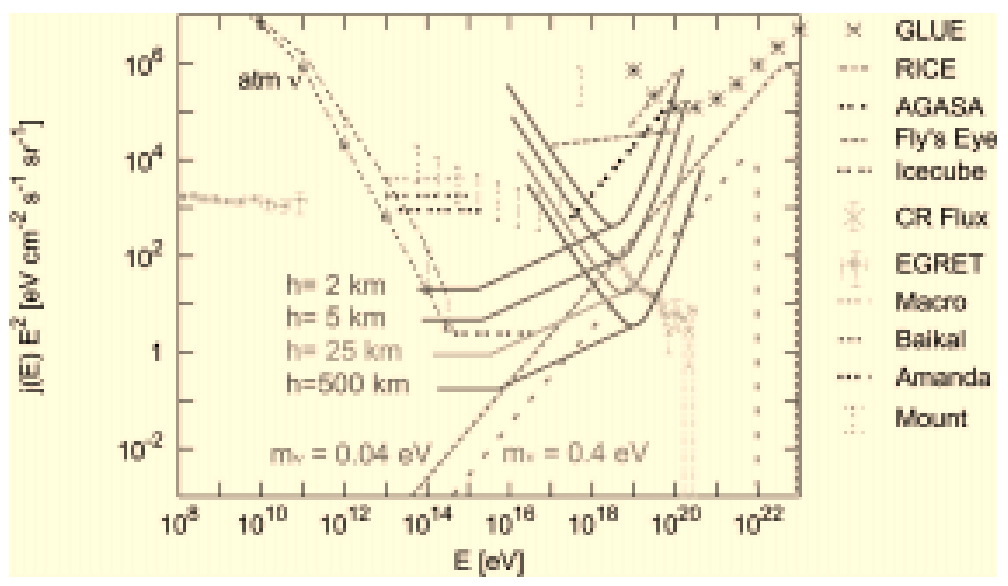

Figure 4: UPTAUs (lower bound on the center) and HORTAUs (right parabolic curves) sensibility at different observer heights $\mathrm{h}(2,5,25,500 \mathrm{~km})$ looking at horizons toward Earth seeking upward Tau Air-Showers adapted over a present neutrino flux estimate in Z-Shower model scenario for light

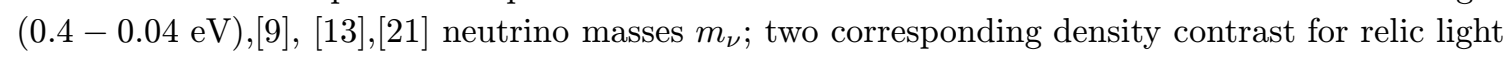
neutrino masses has been assumed; the lower parabolic bound thresholds are at different operation height, in Horizontal (Crown) Detector facing toward most distant horizons edge; these limits are fine tuned (as discussed in the text) in order to receive Tau in flight and its Shower in the vicinity of the detector; we are assuming a duration of data records of a decade comparable to the BATSE record data. The parabolic bounds on the EeV energy range in the right sides are nearly unscreened by the Earth opacity while the corresponding UPTAUs bounds in the center below suffer both of Earth opacity as well as of a consequent shorter Tau interaction lenght in Earth Crust, that has been taken into account.

$1.5 \cdot 10^{-1} \mathrm{~km}^{3}$. The characteristic neutrino interaction are partially summirized in figure isi. The consequent $\tau$ and $\mu$ interactions lenght are also displayed. These lenghts and consequent volume are not extreme. The UPTAUs signal is nearly 15 times larger than the Air-Induced Upward $\nu$ Shower hitting Air. The much wider acceptance of BATSE respect EUSO and the consequent better threshold (in BATSE) is due to the wider angle view of the gamma detector, the absence of any suppression factor as in EUSO duty cycle, as well as the 10 (for BATSE) over 3 (for EUSO) years assumed of record life-time. Any minimal neutrino fluence $\Phi_{\nu_{\tau}}$ of PeVs energetic neutrino: $\Phi_{\nu_{\tau}} \geq 10^{2} \mathrm{eV} \mathrm{cm}^{-2} \mathrm{~s}^{-1}$ might be detectable by EUSO.

\section{Air Induced UHE $\nu$ Shower}

UHE $\nu$ may hit an air nuclei and shower vertically or horizontally or more rarely nearly up-ward: its trace maybe observable by EUSO preferentially in inclined or horizontal case. Indeed Vertical Down-ward $\left(\theta \leq 60^{\circ}\right)$ neutrino induced Air Shower occur mainly at lowest quota and they will only partially shower their UHE $\nu$ energy because of the small slant depth $\left(\leq 10^{3} \mathrm{gcm}^{-2}\right)$ in most vertical down-ward UHE $\nu$ shower. Therefore the observed EUSO air mass $\left(1500 \mathrm{~km}^{3}\right.$, corresponding to a $\sim 150 \mathrm{~km}^{3}$ for $10 \%$ EUSO record time) is only ideally the UHE neutrino calorimeter. Indeed inclined $\sim \theta \geq 60^{\circ}$ ) and horizontal Air-Showers $\left(\sim \theta \geq 83^{\circ}\right)$ (induced by GZK UHE neutrino) may reach their 
maximum output and their event maybe observed ; therefore only a small fraction $(\sim 30 \%$ corresponding to $\sim 50 \mathrm{~km}^{3}$ mass-water volume for EUSO observation) of vertical downward UHE neutrino may be seen by EUSO. This signal may be somehow hidden or masked by the more common down-ward UHECR showers. The key reading signature will be the shower height origination: $(\geq 40 \mathrm{~km})$ for most downward-horizontal UHECR, $(\leq 10 \mathrm{~km})$ for most inclined-horizontal Air UHE $\nu$ Induced Shower. A corresponding smaller fraction $(\sim 7.45 \%)$ of totally Horizontal UHE neutrino Air shower, orphan of their final Cherenkov flash, in competition with the horizontal UHECR, may be also clearly observed: their observable mass is only $V_{A i r-\nu-H o r} \sim 11.1 \mathrm{~km}^{3}$ for EUSO observation duty-cycle. A more rare, but spectacular, double $\nu_{\tau^{-}} \tau$ bang in Air (comparable in principle to the PeVs expected "double bang" in water [3] $\left.\left.\overline{3}_{1}\right]\right)$ may be exciting, but very rare and difficult to be observed.

The EUSO effective calorimeter mass for such Horizontal event is only $10 \%$ of the UHE $\nu$ Horizontal ones $\left(\left(\sim 1.1 \mathrm{~km}^{3}\right)\right)$; therefore its event rate is already almost excluded needing a

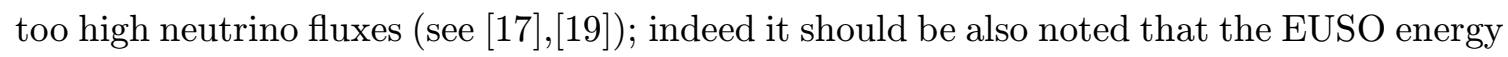
threshold $\left(\geq 3 \cdot 10^{19} \mathrm{eV}\right)$ imply such a very large $\tau$ Lorents boost distance; such large $\tau$ track exceed (by more than a factor three) the EUSO disk Area diameter $(\sim 450 \mathrm{~km})$; therefore the expected Double Bang Air-Horizontal-Induced $\nu$ Shower thresholds are suppressed by a corresponding factor. More abundant single event Air-Induced $\nu$ Shower (Vertical or Horizontal) are facing different Air volumes and quite different visibility. It must be taken into account an additional factor three (for the event rate) (because of three light neutrino states) in the Air-Induced $\nu$ Shower arrival flux respect to incoming $\nu_{\tau}$ (and $\overline{\nu_{\tau}}$ ) in UPTAUs and HORTAUs, making the Air target not totally a negligible calorimeter [1 $\left.{ }_{1}^{1} \hat{9}_{1}^{\prime}\right]$.

There are also a sub-category of $\nu_{\tau}-\tau$ "double bang" due to a first horizontal UHE $\nu_{\tau}$ charged current interaction in air nuclei (the first bang) that is lost from the EUSO view; their UHE secondary $\tau$ fly and decay leading to a Second Air-Induced Horizontal Shower, within the EUSO disk area. These horizontal "Double-Single $\tau$ Air Bang" Showers (or if you like popular terminology, these Air-Earth Skimming neutrinos or just Air-HORTAU event) are produced within a very wide Terrestrial Crown Air Area whose radius is exceeding $\sim 600-800 \mathrm{~km}$ surrounding the EUSO Area of view. However it is easy to show that they will just double the Air-Induced $\nu$ Horizontal Shower rate due to one unique flavour [i, $\left.{ }_{1}^{1}\right]$. Therefore the total Air-Induced Horizontal Shower (for all 3 flavours and the additional $\tau$ decay in flight) are summirized and considered in next figure. The relevant UHE neutrino signal, as discussed below, are due to the Horizontal Tau Air-Showers originated within the (much denser)Earth Crust:the called HORTAUs (or Earth Skimming $\nu_{\tau}$ ).

\section{Horizontal Tau from Earth Skin: HORTAUs}

As already mention the UHE $\nu$ astronomy maybe greatly amplified by $\nu_{\tau}$ appearance via flavour mixing and oscillations. The consequent scattering of $\nu_{\tau}$ on the Mountains or into the Earth Crust may lead to Horizontal Tau Air-Showers :HORTAUs (or so called

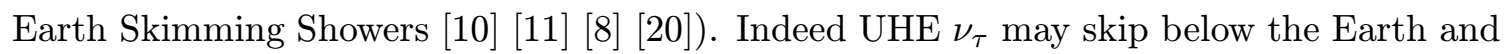




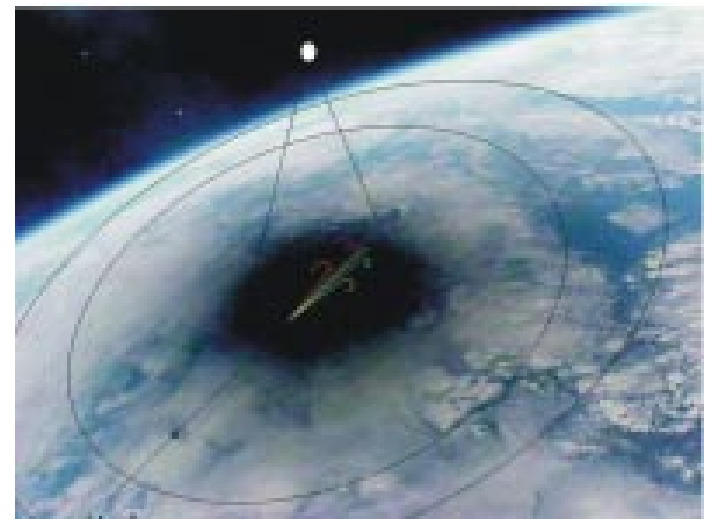

Figure 5: A schematic Horizontal High Altitude Shower or similar Horizontal Tau Air-Shower (HORTAUs) and its open fan-like jets due to geo-magnetic bending seen from a high quota by EUSO satellite. The image background is moon eclipse shadow observed by Mir on Earth. The forked Shower is multi-finger containing a inner $\gamma$ core and external fork spirals due to $e^{+} e^{-}$pairs (first opening) and $\mu^{+} \mu^{-}$pairs.

escape as $\tau$ and finally decay in flight, within air atmosphere, as well as inside the Area of view of EUSO, as shown in Fig. below. Any UHE-GZK Tau Air Shower induced event is approximately born within a wide ring (whose radiuses extend between $R \geq 300$ and $R \leq 800 \mathrm{~km}$ from the EUSO Area center). Because of the wide area and deep $\tau$ penetration

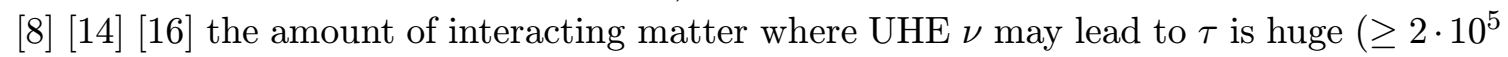
$\mathrm{km}^{3}$ ) ; however only a tiny fraction of these HORTAUs will beam and Shower within the EUSO Area within EUSO. We estimate an effective Volumes for unitary area :

$$
\frac{V_{\text {eff }}}{A_{\oplus}}=\int_{0}^{\frac{\pi}{2}} \frac{\left(2 \pi R_{\oplus} \cos \theta\right) l_{\tau} \sin \theta}{4 \pi R_{\oplus}^{2}} \cdot e^{-\frac{2 R_{\oplus} \sin \theta}{L_{\nu_{\tau}}}} R_{\oplus} d \theta=\frac{1}{2}\left(\frac{L_{\nu_{\tau}}}{2 R_{\oplus}}\right)^{2} l_{\tau} \int_{0}^{\frac{2 R_{\oplus}}{L_{\nu_{\tau}}}} t \cdot e^{-t} d t
$$

Where $V_{\text {eff }}$ is the effective volume where Ultra High Energy neutrino interact while hitting the Earth and lead to escaping UHE Tau: this volume encompass a wide crown belt, due to the cross-section of neutrino Earth skimming along a ring of variable radius $\left(R_{\oplus} \cos \theta\right)$ and a corresponding skin crown of variable depth $l_{\tau} \sin \theta . A_{\oplus}$ is the total terrestrial area, $l_{\tau}$ is the tau interaction lenght, $L_{\nu_{\tau}}$ is the Ultra High Energy Neutrino tau interaction (charged current) in matter. The geometrical quantities are defined in reference

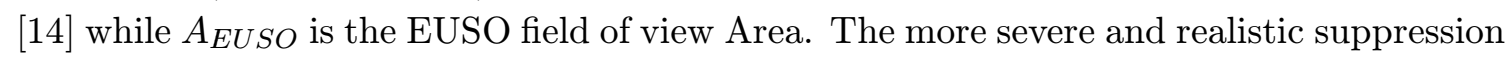
factors should be included in present analytical derivation: first, the exponential decay of air density at highest (derived in Appendix, see reference [8]1); secondly the Earth Crust opacity for UHE neutrino tau at each integral step, already introduced in previous exact $V_{e} f f$ estimate; third, the neutrino energy degradation by neutral current interactions and mainly the charged current production of tau whose energy losses are constraining the detectable volume from EUSO. Assuming two limit cases (Earth all in water or only a thin water layer of terrestrial water no deeper than $4.5 \mathrm{~km}$ in depth ) one finds:

$$
V_{\text {eff-Max }}=\frac{1}{2} A_{\text {Euso }}\left(1-e^{-\frac{L_{0}}{c \tau_{\tau} \gamma_{\tau}}}\right)\left(\frac{L_{\nu_{\tau}}}{2 R_{\oplus}}\right)^{2} \cdot l_{\tau}\left[1-e^{-\frac{2 R_{\oplus}}{L_{\nu_{\tau}}}}\left(1+\frac{2 R_{\oplus}}{L_{\nu_{\tau}}}\right)\right]
$$




$$
V_{\text {eff-Min }}=\frac{1}{2} A_{\text {Euso }}\left(1-e^{-\frac{L_{0}}{c \tau \tau} \gamma_{\tau}}\right)\left(\frac{L_{\nu_{\tau}}}{2 R_{\oplus}}\right)^{2} \cdot l_{\tau}\left[1-e^{-\frac{2 R_{\oplus} \sin \left(\theta_{1}\right)}{L_{\nu_{\tau}}}}\left(1+\frac{2 R_{\oplus} \sin \left(\theta_{1}\right)}{L_{\nu_{\tau}}}\right)\right]
$$

Where $\theta_{1}$ is the opening angle observed at horizon toward the first terrestrial radius step (ocean -inner rock) and it is $\theta_{1} \cong 1.076^{\circ}$.

The effective Volume assuming an outcoming Tau at energy $E_{\tau}=3 \cdot 10^{19} \mathrm{eV}$, becomes $V_{\text {eff }}=5.5 \cdot 10^{2} \mathrm{~km}^{3}$ and the event rate is $N_{e v}=7.7$ in three years [i] $\overline{1}_{-} \bar{j}$. A more accurate estimate offers a slightly lower value. The maximum of the volume occurs at a Tau energy $E_{\tau}=2 \cdot 10^{17} \mathrm{eV}$ and it correspond to a volume $V_{\text {eff }}=7.5 \cdot 10^{3} \mathrm{~km}^{3}$. As it is manifest from the above curve the maximal event numbers takes place at EeV energies. Therefore from here we derived the primary interest for EUSO to seek lowest threshold (as low as $10^{19} \mathrm{eV}$ ). The above expression for the horizontal tau air-shower contains, at lowest energies, the UPTAUs case. Indeed it is possible to see that the same above effective volume at lowest energies simplify and reduces to: $V_{\text {eff }}=\frac{1}{2} A_{\text {Euso }}\left(1-e^{-\frac{L_{0}}{c \tau_{\tau} \gamma_{\tau}}}\right) l_{\tau}$ Because one observes the Earth only from one side the Area factor in eq. 1 should be $A_{\oplus}=2 \pi R_{\oplus}^{2}$ and therefore the half in above formula may be dropped and the final result is just the common expression $V_{\text {eff }}=A_{\text {Euso }} l_{\tau}$.

\section{Conclusions: Event Rate for GZK Neutrinos at EUSO}

The above effective volume should be considered for any given neutrino flux to estimate the outcoming EUSO event number. Here we derive first the analytical formula. These general expression will be plot assuming a minima GZK neutrino flux $\phi_{\nu}$ just comparable to observed UHECR one $\phi_{\nu} \simeq \phi_{U H E C R} \simeq 3 \cdot 10^{-18} \mathrm{~cm}^{-2} \mathrm{~s}^{-1} s r-1$ at the same energy $\left(E_{\nu}=E_{U H E C R} \simeq 10^{19} \mathrm{eV}\right)$. This assumption may changed at will (model dependent) but the event number will scale linearly accordingly to the model. From here we may estimate the event rate in EUSO by a simple extension:

$$
N_{\text {eventi }}=\Phi_{\nu} 4 \pi \eta_{\text {Euso }} \Delta t\left(\frac{V_{e f f}}{L_{\nu}}\right)
$$

Where $\eta_{\text {Euso }}$ is the duty cycle fraction of EUSO, $\eta_{\text {Euso }} \simeq 10 \%, \Delta t \simeq 3$ years and $L_{\nu}$ has been defined in figure above.

$N_{\text {eventi }}=\frac{1}{2} 4 \pi \eta_{\text {Euso }} \Delta t \cdot \Phi_{\nu_{\tau}} A_{\text {Euso }} \cdot\left(1-e^{-\frac{L_{0}}{c \tau_{\tau} \gamma_{\tau}}}\right)\left(\frac{l_{\tau}}{L_{\nu_{\tau}}}\right)\left(\frac{L_{\nu_{\tau}}}{2 R_{\oplus}}\right)^{2} \cdot\left[1-e^{-\frac{2 R_{\oplus}}{L_{\nu_{\tau}}}}\left(1+\frac{2 R_{\oplus}}{L_{\nu_{\tau}}}\right)\right]$

It should be remind that all these event number curves for EUSO are already suppressed by a factor $\eta_{\text {Euso }}=0.1$ due to minimal EUSO duty cycle.

However the Air-Shower induced neutrino may reflect all three light neutrino flavours, while HORTAUs are made only by $\nu_{\tau}, \overline{\nu_{\tau}}$ flavour. Nevertheless the dominant role of HORTAUs overcome (by a factor $\geq 6$ ) all other Horizontal EUSO neutrino event at lowest energy edges $10^{19} \mathrm{eV}$ : their expected event rate are, at $\Phi_{\nu} \geq 3 \cdot 10^{3} \mathrm{eV} \mathrm{cm}^{-2} \mathrm{~s}^{-1}$ neutrino fluence, 


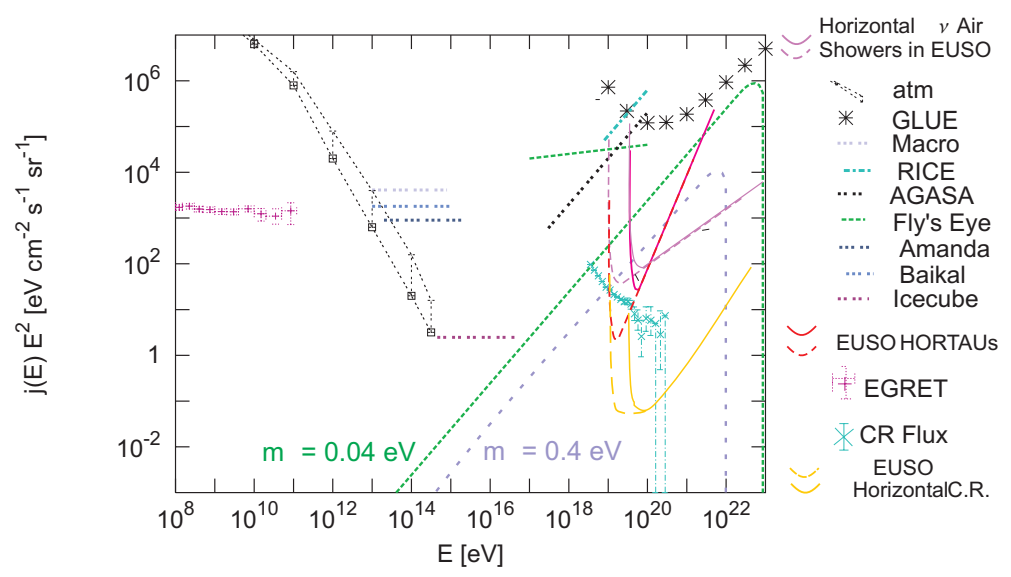

Figure 6: EUSO thresholds for Horizontal Tau Air-Shower shower, HORTAUs (or Earth Skimming Showers) over all other $\gamma, \nu$ and Cosmic Rays (C.R.) Fluence and bounds. The Fluence threshold for EUSO has been estimated for a three year experiment lifetime. Competitive experiment are also shown as well as the Z-Shower expected spectra in light neutrino mass values $\left(m_{\nu}=0.04,0.4\right.$

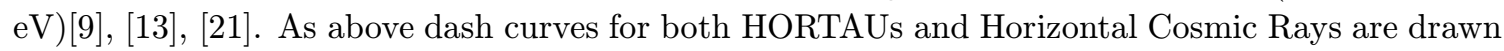
assuming an EUSO threshold at $10^{19} \mathrm{eV}$.

as in Z-Shower model, a few hundred event a year and they may already be comparable or even may exceed the expected Horizontal CR rate [1] $\left.{ }_{1}^{1}\right]$. Dash curves for both HORTAUs and Horizontal Cosmic Rays are drawn assuming an EUSO threshold at $10^{19} \mathrm{eV}$. Because the bounded $\tau$ flight distance (due to the contained terrestrial atmosphere height) the main signal is better observable at $1.1 \cdot 10^{19} \mathrm{eV}$ than higher energies as emphasized at different curves in figure above. It should be noticed that HORTAUs are very long high altitude showers. Their lenght may exceed two hundred kilometers. This trace may be larger than the EUSO radius of Field of View. Therefore there may be both contained and partially contained events. There may be also crossing HORTAUs at the edges of EUSO disk area. However most of the events will be partially contained, either just on their birth or at their end, equally balanced in number. Because of the HORTAU Jet forked shower, its up-going direction, its fan like structure (see Fig. be the manifest and mostly useful and clear event. The area of their origination, four times larger than EUSO field of view, will be mostly outside the same EUSO area. Their total number count will double the event rate $N_{e v}$ (and the corresponding $V_{\text {eff }}$ ) of HORTAUs [1] 1 in]. The additional crossing event will make additional events (a small fraction) of the effective volume of HORTAUs at $10^{19} \mathrm{eV}$ the most rich neutrino signal few times larger the Air induced events. The same doubling will apply only to UHECR horizontal shower while the downward Ultra High Energy Neutrino will not share this phenomena (out of those $\simeq 6 \%$ of the Horizontal Air Neutrino Shower). Even for the most conservative scenario where a minimal GZK- $\nu$ fluence must take place (at least at: $E_{\nu} \Phi_{\nu} \simeq 30 \mathrm{eVcm}^{-2} \mathrm{~s}^{-1} \mathrm{sr}^{-1}$, just comparable to well observed Cosmic Ray fluence), half a dozen of such UHE astrophysical neutrino must be observed during three year of EUSO data recording. These HORTAUs will be not be observable by other competitive experiment as AUGER. There- 


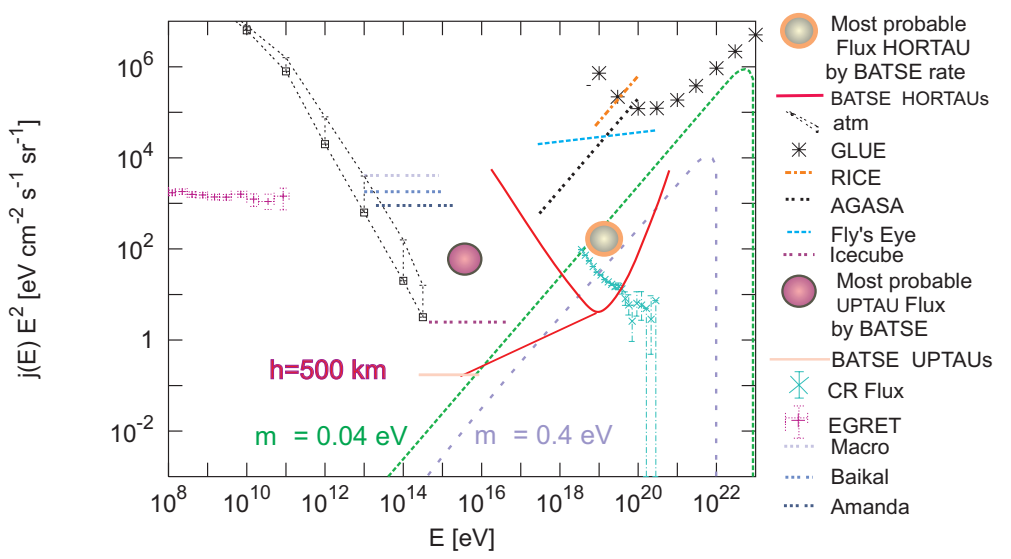

Figure 7: Neutrino Flux derived by BATSE Terrestrial Gamma Flashes assuming them as $\gamma$ secondaries of upward Tau Air-showers. These fluxes are estimated using the data from Terrestrial Gamma Flash (1991-2000) normalized during their most active trigger and TGF hard activities. The UPTAUs and HORTAUs rate are normalized assuming that the events at geo-center angle above $50^{\circ}$ might be of HORTAU nature.

fore to improve the HORTAU visibility in EUSO one must: a) Improve the fast pattern recognition of Horizontal Shower Tracks with their few distant dots with forking signature, b) Enlarge the Telescope Radius to embrace also lower $10^{19} \mathrm{eV}$ energy thresholds where UHE HORTAU neutrino signals are enhanced, c) Consider a detection at angular $\Delta \theta$ and at height $\Delta h$ level within an accuracy $\Delta \theta \leq 0.2^{\circ}, \Delta h \leq 2 \mathrm{~km}$. Even all the above results

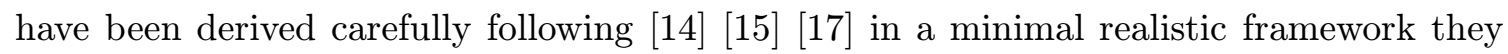
may be used within $20 \%$ nominal value due to the present uncertain in EUSO detection capabilities. In conclusion UHECR and Neutrino Astronomy face a new birth. The Neutrino Astronomy may be widely discovered by Upward and Horizontal $\tau$ Air-Showers. The Tau neutrinos, born abundantly by flavour mixing will probe such Astronomy above PeVs up to EeVs energies, where astrophysics rule over atmospheric neutrino noise. The same UHE $\overline{\nu_{e}}$ at $E_{\nu_{e}}=\frac{M_{W}{ }^{2}}{2 \cdot m_{e}} \simeq 6.3 \mathrm{PeV}$ must be a peculiar neutrino astronomy born beyond Mountain

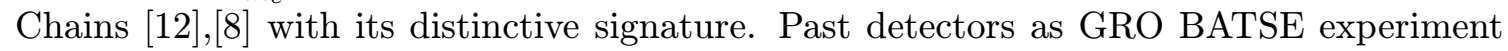
might already found some direct signals of such rare UPTAUs or HORTAUs; indeed their observed Terrestrial Gamma Flash event rate translated into a neutrino induced upward shower (see Fig, $\overline{7}_{1}$ ) leads to a most probable flux both at PeVs energies just at a level comparable to most recent AMANDA threshold sensitivity: for horizontal TGF events at $10^{19}$ $\mathrm{eV}$ windows, the signals fit the Z-Burst model needed fluence (for neutrino at $0.04-0.4$

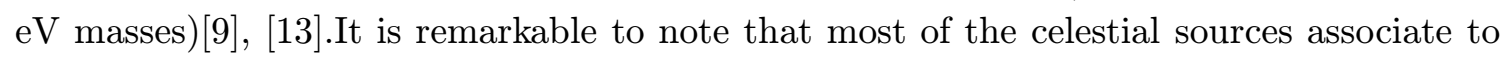
TGF are on the galactic plane and in particular toward the galactic center (namely black hole candidate source $G X 339-4$ and most active known galactic sources [iَ EUSO telescope detector, if little enlarged will easily probe even the smallest, but necessary Neutrino GZK fluxes with clear sensitivity (see Fig. $\overline{\underline{6}}_{i}$ ). We therefore expect that a serial of experiment will soon turn toward this last and neglected, but most promising Highest Energy Tau Neutrino Astronomy searching for GZK or Z-Showers neutrino signatures. 


\section{References}

[1] Becattini F and Bottai S 2001 Astropart. Phys. 15323

[2] SNO Collaboration, 2002, Phys. Rev. Lett. 89 (2002) 011302.

[3] Bertou X, et al. 2002 Astropart. Phys. 17 183-193

[4] Dolgov A 2002 Phys. Rept. 370, 333-535; 2002 hep-ph/0202122

[5] Dutta Iyer S, Reno M H, et al. 2001 Phys. Rev. D63 094020

[6] Fargion D and Salis A 1997 Proc.25th ICRC HE-4-6 153

[7] Fargion D, Mele B and Salis A, Ap. J. 517 (1999) 725; astro-ph/9710029

[8] Fargion D, Ap. J. 570 (2002) 909-925, astro-ph/0002453; astro-ph/9704205

[9] Fargion D and Grossi M, De Sanctis Lucentini P G, Di Troia C and Konoplich R V; Dark2000, Heidelberg 10-14, July, Ed. Klapdor-Kleingrothaus H V, Springer 2001 p. 455-468

[10] Fargion D 2001 27th ICRC 2001 HE2.5 1297-1300 2001 astro-ph/0106239

[11] Fargion D 2001 27th ICRC 2001 HE1.8 Germany 903-906 2001 astro-ph/0107094

[12] Fargion D, Aiello A ,Conversano R, 26th ICRC HE6.1.10 396-398; 1999; astro-ph/9906450

[13] Fargion D, Grossi M, De Sanctis Lucentini P G and Troia C J 2001 Phys. Soc. Jpn. 70 46-57

[14] Fargion D 2002 hep-ph/0206010.

[15] Fargion D 2002 SPIE Conference 4858-01 2002 hep-ph/0208093

[16] Fargion D 2002 Oulu, Beyond the Standard Model Conference 2002, Ed. H.V. Klapdor-Kleingrothaus, IOP, Bristol (2003) 543-567, and hep-ph/0211153

[17] Fargion D., Khlopov M, Konoplich R, De Sanctis Lucentini P.G., De Santi M.s, Mele B. astro-ph/0303233

[18] Fargion D,M.Khlopov, R.Konoplich, De Sanctis Lucentini P.G., De Santis M., B. Mele. 2002, Research Sigh Point, Recent Research Developments Astrophysics 1-58, 2003;

[19] Fargion D, De Sanctis Lucentini P.G., De Santis M. 2002, hep-ph/0305128; Fargion D., astro-ph/0307485.

[20] Feng J L, Fisher P, Wilczek F and Terri M Yu hep-ph/0105067

[21] Fodor Z., Katz S. D. and Ringwald A. 2002b JHEP 0206 (2002) 046

[22] Fukuda Y et al. [Super-Kamiokande Collaboration] 1998 Phys. Rev. Lett. 811562

[23] Gallex Collaboration, 1992, Phys.Letter.B 285 (1992) 376.

[24] Gorbunov D S, Tinyakov P G ,Tkachev I I and Troitsky S V 2002 astro-ph/0204360 and 2002 Astrophys.J. 577 L93

[25] Greisen K 1966 Phys. Rev. Lett. 16748

[26] Hayashida N et al. AGASA collaboration 1999 Astropart. Phys. 10 303-311

[27] Kalashev O E, Kuzmin V A, Semikoz D V and Sigl G 2002 Phys. Rev. D 66063004

[28] KamLand Collaboration, 2002, hep-ex/0212021 
[29] Klapdor-Kleingrothaus H V, Dietz A, Harney H L and Krivosheina I V 2001 Mod. Phys. Lett. A 16 2409;

[30] Halzen F and Saltzberg D 1998 Phys. Rev. Lett. 81 4305-4308

[31] Hou G W S and Huang M A 2002 astro-ph/0204145

[32] Gandhi R, Quigg C, Reno M H and Sarcevic I 1998 Phys. Rev. D 58093009

[33] Learned J G and Pakvasa S 1995 Astropart. Phys. 3267

[34] Yoshida S, Sigl G and Lee S 1998 Phys. Rev. Lett. 815505

[35] Weiler T 1999 Astropart. Phys. 11303

[36] Zatsepin G T and Kuzmin V A 1966 JETP Lett. 478 